\title{
BMJ Open Use of the first National Early Warning Score recorded within 24 hours of admission to estimate the risk of in- hospital mortality in unplanned COVID-19 patients: a retrospective cohort study
}

Donald Richardson, ${ }^{1}$ Muhammad Faisal (D) , ${ }^{2,3,4,5}$ Massimo Fiori, ${ }^{6}$ Kevin Beatson, ${ }^{6}$ Mohammed Mohammed ${ }^{2,7}$

To cite: Richardson D, Faisal M, Fiori $\mathrm{M}$, et al. Use of the first National Early Warning Score recorded within 24 hours of admission to estimate the risk of in-hospital mortality in unplanned COVID-19 patients: a retrospective cohort study. BMJ Open 2021;11:e043721. doi:10.1136/ bmjopen-2020-043721

- Prepublication history and additional materials for this paper is available online. To view these files, please visit the journal online (http://dx.doi. org/10.1136/bmjopen-2020043721).

Received 12 August 2020 Revised 12 January 2021 Accepted 14 January 2021

D) Check for updates

(c) Author(s) (or their employer(s)) 2021. Re-use permitted under CC BY-NC. No commercial re-use. See rights and permissions. Published by BMJ.

For numbered affiliations see end of article.

\section{Correspondence to} Dr Mohammed Mohammed; m.a.mohammed5@bradford. ac.uk

\section{ABSTRACT}

Objectives Although the National Early Warning Score (NEWS) and its latest version NEWS2 are recommended for monitoring deterioration in patients admitted to hospital, little is known about their performance in COVID-19 patients. We aimed to compare the performance of the NEWS and NEWS2 in patients with COVID-19 versus those without during the first phase of the pandemic.

Design A retrospective cross-sectional study.

Setting Two acute hospitals (Scarborough and York) are combined into a single dataset and analysed collectively. Participants Adult ( $\geq 18$ years) non-elective admissions discharged between 11 March 2020 and 13 June 2020 with an index or on-admission NEWS2 electronically recorded within \pm 24 hours of admission to predict mortality at four time points (in-hospital, 24 hours, 48 hours and 72 hours) in COVID-19 versus non-COVID-19 admissions.

Results Out of 6480 non-elective admissions, 620 (9.6\%) had a diagnosis of COVID-19. They were older (73.3 vs 67.7 years), more often male ( $54.7 \%$ vs $50.1 \%$ ), had higher index NEWS (4 vs 2.5 ) and NEWS2 (4.6 vs 2.8) scores and higher in-hospital mortality (32.1\% vs $5.8 \%)$. The cstatistics for predicting in-hospital mortality in COVID-19 admissions was significantly lower using NEWS (0.64 vs 0.74 ) or NEWS2 (0.64 vs 0.74$)$, however, these differences reduced at 72 hours (NEWS: 0.75 vs 0.81 ; NEWS2: 0.71 vs 0.81 ), 48 hours (NEWS: 0.78 vs 0.81 ; NEWS2: 0.76 vs 0.82 ) and 24hours (NEWS: 0.84 vs 0.84 ; NEWS2: 0.86 vs 0.84$)$. Increasing NEWS2 values reflected increased mortality, but for any given value the absolute risk was on average $24 \%$ higher (eg, NEWS2 $=5$ : $36 \%$ vs $9 \%$ ).

Conclusions The index or on-admission NEWS and NEWS2 offers lower discrimination for COVID-19 admissions versus non-COVID-19 admissions. The index NEWS2 was not proven to be better than the index NEWS. For each value of the index NEWS/NEWS2, COVID-19 admissions had a substantially higher risk of mortality than non-COVID-19 admissions which reflects the increased baseline mortality risk of COVID-19.
Strengths and limitations of this study

- This study data is from a single National Health Service Trust and used the index National Early Warning Score (NEWS)/NEWS2 scores. The extent to which these findings are generalisable, especially to minority ethnic groups with higher mortality, require further study.

- Although we found no evidence of NEWS2 as having a superior performance to NEWS, this does suggest that the additional enhancements in NEWS2 are having a limited impact and the underlying reasons need further study.

- NEWS and NEWS2 are repeatedly updated for each patient according to local hospital protocols, and the extent to which changes in NEWS or NEWS2 over time reflect changes in mortality risk needs further study.

\section{INTRODUCTION}

The novel coronavirus SARS-CoV-2, which was declared as a pandemic on 11 March 2020, produces the newly identified disease 'COVID-19' in patients with symptoms (Coronaviridae Study Group of the International Committee on Taxonomy of Viruses ${ }^{1}$ ) which has challenged healthcare systems worldwide.

Patients with COVID-19 admitted to hospital can develop severe disease with life threatening respiratory and/or multiorgan failure $^{23}$ with a high risk of mortality in part due to the lack of effective treatment for the underlying disease in the early phase of the pandemic. Thus, it is recommended that patients at risk of deterioration are referred to critical care. The appropriate early assessment and management of patients with COVID-19 is important in ensuring high-quality care. 
In the UK National Health Service (NHS), the patient's vital signs are monitored and summarised into a National Early Warning Score (NEWS) or its latest iteration, NEWS2. ${ }^{4}$ NEWS is used across the world. ${ }^{4}$ NEWS and NEWS2 are calculated from six physiological variables or vital signs-respiration rate, oxygen saturation, temperature, systolic blood pressure, heart rate and level of consciousness (alert, confusion, voice, pain, unresponsive) and also use of supplemental oxygen-routinely collected by nursing staff as an integral part of the process of care, usually for all patients, and then repeated thereafter depending on local hospital protocols. NEWS2 includes two oxygen saturation scales (scale 1 and scale 2) and new confusion. ${ }^{5}$ NEWS2 points are allocated according to these clinical observations. A higher NEWS2 correlates with a higher chance of deterioration. Gidari et $a t^{6}$ evaluated NEWS2 at hospital admission of patients with COVID-19 as a predictor of intensive care unit (ICU) admission. Furthermore, Kostakis $e t a l^{7}$ investigated association of the last or ultimate recorded NEWS2/NEWS within 24 hours of death or ICU admission in COVID-19 and non-COVID cohorts.

Although NEWS2 is recommended for clinical use in patients with COVID- $19,{ }^{8}$ little is known about how NEWS2 performs in practice. In this study, we aimed to compare the performance of NEWS and NEWS2 via unplanned admissions to a teaching hospital during the first phase of the novel coronavirus SARS CoV-2 (COVID19) pandemic, in predicting in-hospital mortality at four time points ( 24 hours, 48 hours, 72 hours and in-hospital mortality) in COVID-19 versus non-COVID-19 admissions. For all our analyses, we use the on-admission or index NEWS2/NEWS because this is an early indicator of the severity of illness.

\section{METHODS}

\section{Setting and data}

Our cohort of unplanned admissions are from two acute hospitals which are approximately $65 \mathrm{~km}$ apart in the Yorkshire and the Humber region of England-Scarborough hospital ( $\mathrm{n} 300$ beds) and York Hospital ( $\mathrm{n} 700$ beds), managed by York Teaching Hospitals NHS Foundation Trust. For the purposes of this study, the two acute hospitals are combined into a single dataset and analysed collectively. The hospitals have electronic NEWS2 scores and vital signs recording which are routinely collected as part of the patient's process of care.

We considered all adult (age $\geq 18$ years) emergency medical admissions (non-elective/unplanned excluding ambulatory care area patients), discharged during 3 months (11 March 2020-13 June 2020), with electronic NEWS2 recorded within \pm 24 hours of admission. For each emergency admission, we obtained a pseudonymised patient identifier, patient's age (years), sex (male/female), ethnicity, body mass index (BMI kg/m²), discharge status (alive/dead), admission and discharge date and time, diagnoses codes based on the 10th revision of the International Statistical Classification of Diseases (ICD-10), ${ }^{910}$ NEWS2 (including its subcomponents respiratory rate, temperature, systolic pressure, pulse rate, oxygen saturation, oxygen supplementation, oxygen scales 1 and 2, and alertness including confusion). ${ }^{45}$ The diastolic blood pressure was recorded at the same time as systolic blood pressure. Historically, diastolic blood pressure has always been a routinely collected physiological variable on vital sign charts and is still collected where electronic observations are in place (see online supplemental tables S1 and S2). NEWS2 produces integer values that range from 0 (indicating the lowest severity of illness) to 20 (the maximum NEWS2 value possible). The index NEWS2 was defined as the first electronically recorded NEWS2 within \pm 24 hours of the admission time as vital signs can be collected before admission. We excluded records where the first NEWS2 was not within \pm 24 hours of admission or was missing/not recorded (see table 1). Since NEWS2 extends NEWS, we used the same dataset to compare NEWS and NEWS2 especially as NEWS is still in widespread use. The ICD-10 code 'U071' was used to identify records with COVID-19. We searched, primary and secondary ICD-10 codes for ' $\mathrm{U} 071$ ' for identifying COVID-19.

\section{Statistical modelling}

We began with exploratory analyses including line plots that showed the relationship between age, vital signs, NEWS2/NEWS and risk of in-hospital death in COVID-19 and non-COVID-19. We compared the continuous covariates using a two-sample independent t-test (for normal data) or Wilcoxon rank-sum test (for non-normal data). We compared the categorical covariates using a $\chi^{2}$ proportion test. $\mathrm{P}$ values less than 0.05 were defined as statistically significant.

We determined the discrimination of NEWS and NEWS2 using the concordance or c-statistic which is interpreted as the probability that a deceased patient had a higher risk of death than a randomly chosen nondeceased patient. For a binary outcome (alive/died), the c-statistic is the area under the Receiver Operating Characteristics (ROC) curve. ${ }^{11}$ The ROC curve is a plot of the sensitivity, (true positive rate), vs 1-specificity, (false positive rate), for consecutive predicted risks. A c-statistic of 0.5 is no better than tossing a coin, while a perfect model has a c-statistic of 1 . In general, values less than 0.7 are considered to show poor discrimination, values of $0.7-0.8$ can be described as reasonable, and values above 0.8 suggest good discrimination. ${ }^{12}$ We developed two separate logistic regression models for predicting in-hospital mortality with NEWS and NEWS2 as covariates, respectively. We assessed the performance of the index NEWS or index NEWS2 in predicting the mortality at four specified time points-24 hours, 48 hours, 72 hours and in-hospital in COVID-19 and non-COVID-19 patients using the c-statistic. For each time point we used the index or on-admission NEWS2/NEWS score. 


\begin{tabular}{|c|c|c|c|}
\hline Characteristic & COVID-19 & $\begin{array}{l}\text { Non- } \\
\text { COVID-19 }\end{array}$ & $\begin{array}{l}P \\
\text { value }\end{array}$ \\
\hline $\mathrm{N}$ & 620 & 5824 & - \\
\hline Male (\%) & $339(54.7)$ & $2918(50.1)$ & 0.033 \\
\hline Mean age (years) (SD) & $73.3(15.4)$ & $67.7(19)$ & $<0.001$ \\
\hline Admission type & & & $<0.001$ \\
\hline Medical & $588(94.8)$ & $4727(81.1)$ & \\
\hline Surgical & $32(5.2)$ & 1097 (18.9) & \\
\hline Ethnicity & & & $<0.001$ \\
\hline White & $465(75)$ & $4668(80.2)$ & \\
\hline $\begin{array}{l}\text { Black, Asian and other } \\
\text { minority ethnic }\end{array}$ & $34(5.5)$ & $152(2.6)$ & \\
\hline Missing & $121(19.5)$ & $1004(17.2)$ & \\
\hline Median BMI $(\mathrm{IQR})^{\star}\left(\mathrm{kg} / \mathrm{m}^{2}\right)$ & $27.5(8.4)$ & $26(7.6)$ & $<0.001$ \\
\hline Mean NEWS (SD) & $4(2.8)$ & $2.5(2.3)$ & $<0.001$ \\
\hline Mean NEWS2 (SD) & $4.6(3)$ & $2.8(2.7)$ & $<0.001$ \\
\hline \multicolumn{4}{|l|}{ Vital signs } \\
\hline $\begin{array}{l}\text { Mean Respiratory rate } \\
\text { (breaths per minute) (SD) }\end{array}$ & $23.5(6.6)$ & $19.8(5)$ & $<0.001$ \\
\hline $\begin{array}{l}\text { Mean temperature }\left({ }^{\circ} \mathrm{C}\right) \\
(\mathrm{SD})\end{array}$ & $36.8(1.1)$ & $36.3(0.9)$ & $<0.001$ \\
\hline $\begin{array}{l}\text { Mean systolic pressure } \\
(\mathrm{mm} \mathrm{Hg})(\mathrm{SD})\end{array}$ & $136.1(25.8)$ & $142.5(29.2)$ & $<0.001$ \\
\hline $\begin{array}{l}\text { Mean diastolic pressure } \\
(\mathrm{mm} \mathrm{Hg})(\mathrm{SD})\end{array}$ & $76.5(16.3)$ & $79.4(16.8)$ & $<0.001$ \\
\hline $\begin{array}{l}\text { Mean pulse rate (beats } \\
\text { per minute) (SD) }\end{array}$ & $92.2(22.1)$ & $88.5(22.2)$ & $<0.001$ \\
\hline $\begin{array}{l}\text { Mean \% oxygen } \\
\text { saturation (SD) }\end{array}$ & $94.8(4.4)$ & $96.4(2.9)$ & $<0.001$ \\
\hline $\begin{array}{l}\text { Oxygen supplementation } \\
(\%)\end{array}$ & $207(33.4)$ & $667(11.5)$ & $<0.001$ \\
\hline $\begin{array}{l}\text { Mean oxygen flow rate } \\
\text { (litre per minute) (SD) }\end{array}$ & $7.6(5.8)$ & $6.4(5.5)$ & 0.008 \\
\hline Oxygen scale 2 (\%) & $42(6.8)$ & $361(6.2)$ & 0.634 \\
\hline Alertness & & & $<0.001$ \\
\hline Alert (\%) & 514 (82.9) & $5239(90)$ & \\
\hline Baseline confusion (\%) & $5(0.8)$ & $45(0.8)$ & \\
\hline New confusion (\%) & $19(3.1)$ & $82(1.4)$ & \\
\hline Pain (\%) & $0(0)$ & $49(0.8)$ & \\
\hline Voice (\%) & $58(9.4)$ & 227 (3.9) & \\
\hline Unconscious (\%) & $24(3.9)$ & $182(3.1)$ & \\
\hline $\begin{array}{l}\text { Referred to critical care } \\
\text { outreach team (\%) }\end{array}$ & $91(14.7)$ & $211(3.6)$ & $<0.001$ \\
\hline Admission to ICU (\%) & $42(6.8)$ & $147(2.5)$ & $<0.001$ \\
\hline Palliative care (\%) & 65 (10.5) & $288(4.9)$ & $<0.001$ \\
\hline On ventilation (\%) & $18(2.9)$ & $12(0.2)$ & $<0.001$ \\
\hline $\begin{array}{l}\text { Median length of stay } \\
\text { (days) (IQR) }\end{array}$ & $7.3(11.7)$ & $3(5.5)$ & $<0.001$ \\
\hline $\begin{array}{l}\text { Mortality within } 24 \text { hours } \\
(\%)\end{array}$ & $9(1.5)$ & $53(0.9)$ & 0.273 \\
\hline
\end{tabular}

Table 1 Characteristics of emergency medical admissions in COVID-19 versus non-COVID-19

Continued
Table 1 Continued

\begin{tabular}{|c|c|c|c|}
\hline Characteristic & COVID-19 & $\begin{array}{l}\text { Non- } \\
\text { COVID-19 }\end{array}$ & $\begin{array}{l}P \\
\text { value }\end{array}$ \\
\hline $\begin{array}{l}\text { Mortality within } 48 \text { hours } \\
(\%)\end{array}$ & $15(2.4)$ & $94(1.6)$ & 0.189 \\
\hline $\begin{array}{l}\text { Mortality within } 72 \text { hours } \\
(\%)\end{array}$ & $33(5.3)$ & $131(2.3)$ & $<0.001$ \\
\hline In-hospital mortality & 199 (32.1) & $336(5.8)$ & $<0.001$ \\
\hline
\end{tabular}

${ }^{*} \mathrm{BMI}$ is missing 188 (30.3\%) for COVID and 2283 (39.2\%) for non-COVID.

BMI, body mass index; ICU, intensive care unit; NEWS, National Early Warning Score.

We assessed the sensitivity, specificity, positive predictive value, negative predictive value and likelihood ratios for NEWS and NEWS2 at values $\geq 5$ which is the usual threshold value for escalation to critical care which equates to a $13 \%$ mortality risk under NEWS and an $11 \%$ risk under NEWS2. The 95\% CI for the c-statistic was derived using DeLong's method as implemented in the pROC library ${ }^{13}$ in R. ${ }^{14}$ We followed the Strengthening the Reporting of Observational Studies in Epidemiology guidelines to report the findings. ${ }^{15}$ All analyses were undertaken using $R^{14}$ and Stata. ${ }^{16}$

\section{Patient and public involvement}

There was no patient involvement in this study.

\section{RESULTS}

\section{Cohort description}

There were 6480 discharges over 3 months. We excluded $36(0.6 \%)$ records because the index NEWS2 was not recorded within \pm 24 hours of the admission date/time or NEWS2 was missing or not recorded at all (see online supplemental table S3).

We analysed data from 6444 admissions, of which $9.6 \%(620 / 6444)$ were diagnosed COVID-19. The demographic, vital signs and outcome profiles of the COVID-19 vs non-COVID-19 admissions is shown in table 1 and online supplemental figure S1. COVID-19 admissions were older (73.3 vs $67.7, \mathrm{p}<0.001)$, more likely to be male $(54.7 \%$ vs $50.1 \%, \mathrm{p}<0.001)$, with higher BMI $\left(\mathrm{kg} / \mathrm{m}^{2}\right)(27.5$ vs 26 , $\mathrm{p}<0.001)$ than non-COVID-19 admissions. Furthermore, they had higher index NEWS (4.0 vs $2.5, \mathrm{p}<0.001)$ and index NEWS2 (4.6 vs 2.8, $\mathrm{p}<0.001)$ than non-COVID-19 admissions which was reflected in differences in vital signs notably, a higher respiratory rate (23.5 vs $19.8, \mathrm{p}<0.001)$, lower oxygen saturation $(94.8 \%$ vs $96.4 \%, \mathrm{p}<0.001)$, higher frequency of oxygen supplementation $(33.4 \%$ vs $11.5 \%, \mathrm{p}<0.001)$, lower systolic blood pressure $(136.1 \mathrm{~mm}$ $\mathrm{Hg}$ vs $142.5 \mathrm{~mm} \mathrm{Hg}, \mathrm{p}<0.001)$ and less likely to be alert $(82.9 \%$ vs $90 \%, \mathrm{p}<0.001)$.

COVID-19 admissions were more likely to be referred to the critical care outreach team $(14.7 \%$ vs $3.6 \%, \mathrm{p}<0.001)$, admitted to the ICU (6.8\% vs $2.5 \%)$ and referred to palliative care $(10.5 \%$ vs $4.9 \%)$. They also had longer hospital 

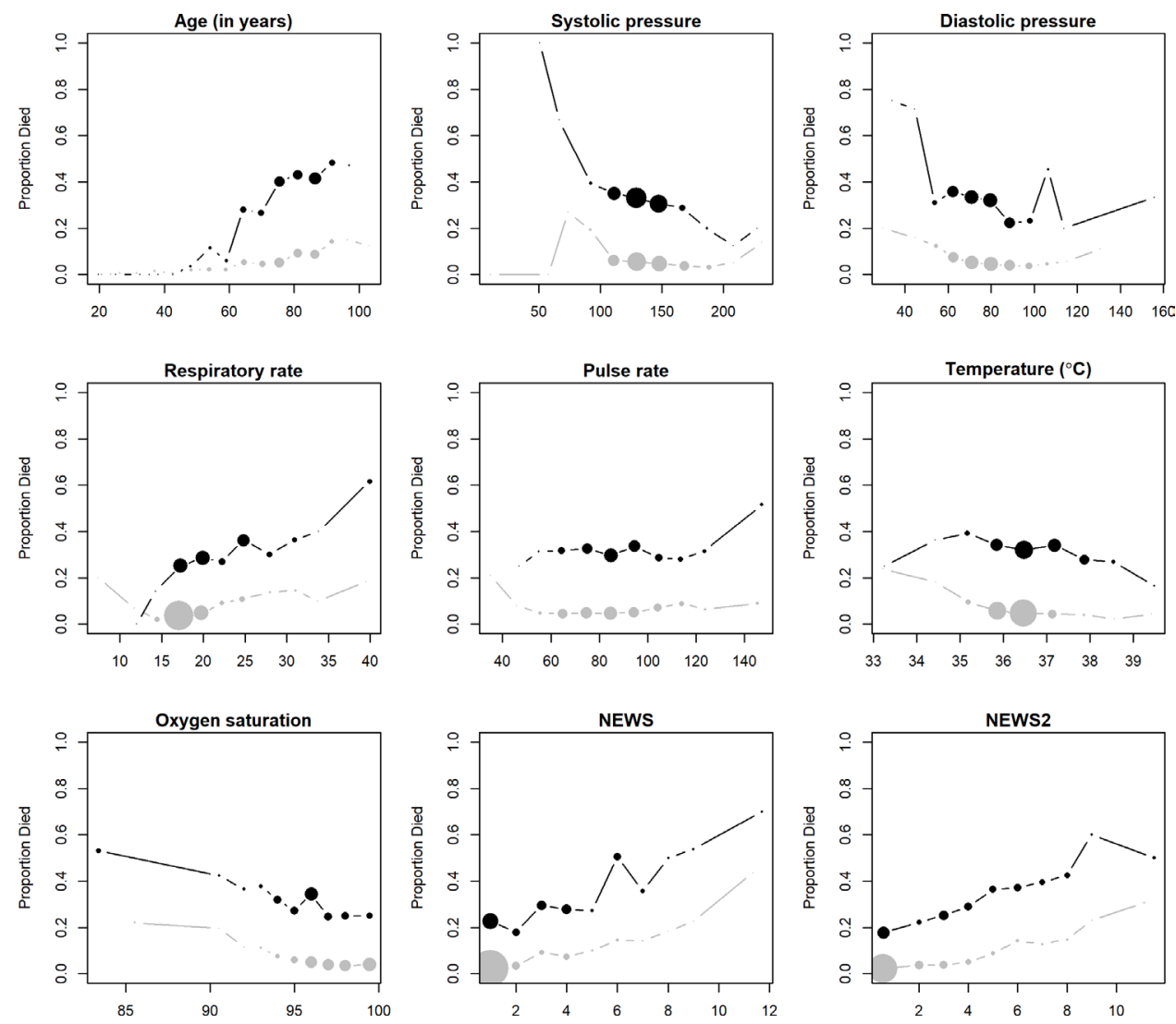

Figure 1 Line plots showing the observed risk of in-hospital mortality with continuous covariates for COVID-19 (black colour) and non-COVID-19 (grey colour) admissions. Size of circles reflects sample size independently in the COVID-19 and nonCOVID-19 groups. NEWS, National Early Warning Score.

stay (7.3 days vs 3.0 days, $\mathrm{p}<0.001)$ and higher in-hospital mortality $(32.1 \%$ vs $5.8 \%, \mathrm{p}<0.001)$.

Figure 1 shows the relationship between continuous covariates and the observed risk of in-hospital mortality in COVID-19 versus non-COVID-19 admissions. While the pattern of mortality was broadly similar between COVID-19 and non-COVID-19 admissions, COVID-19 admissions had a consistently higher risk of mortality for the range of covariate values (see figure 1 and online supplemental figure S2). Figure 1 also shows that although increasing NEWS and NEWS2 scores reflected increased mortality, but for any given value of NEWS or NEWS2 the risk of mortality for COVID-19 was on average $24 \%$ higher and at a NEWS or NEWS2 of 5 the risk of mortality in COVID-19 vs non-COVID-19 was $36 \%$ vs $9 \%$.

The performance of index NEWS2 to predict the risk of death (24 hours, 48 hours, 72 hours, in-hospital) in COVID-19 and non-COVID-19 admissions is shown in figure 2 and online supplemental tabe S4. The c-statistics for predicting in-hospital mortality in COVID-19 admissions was significant lower than for patients without COVID-19 (NEWS: 0.64 vs 0.74; NEWS2: 0.64 vs 0.74), however, these differences reduced at 72 hours (NEWS: 0.75 vs 0.81 ; NEWS2: 0.71 vs 0.81 ), 48 hours (NEWS: 0.78 vs 0.81 ; NEWS2: 0.76 vs 0.82 ) and 24hours (NEWS: 0.84 vs 0.84 ; NEWS2: 0.86 vs 0.84 ). We found the same performance for medical and surgical admissions (see online supplemental table S5). Online supplemental figure S3 shows Kaplan-Meier curve for mortality for COVID-19 and non-COVID-19 admissions.

Table 2 includes the sensitivity, specificity, positive and negative predictive values for NEWS and NEWS2 for COVID-19 and non-COVID-19 patients. NEWS2 had higher sensitivity but lower specificity compared with NEWS.

\section{DISCUSSION}

While NEWS and NEWS2 are recommended for monitoring patients with COVID-19, we found that the index or on-admission NEWS/NEWS2 offered lower discrimination for COVID-19 patients versus non-COVID-19 patients. We also found that the index NEWS2 was not better than index NEWS. For each value of the index NEWS/NEWS2, COVID-19 patients had a substantially higher risk of in-hospital mortality than non-COVID-19 patients, which equated to an average $24 \%$ risk difference which reflects the higher baseline risk of mortality in our COVID-19 patients. However, the c-statistics for the index NEWS/NEWS2 improved with shorter time horizons with the highest discrimination (above 0.8) being seen for predicting mortality risk within 24hours of the index NEWS/NEWS2. 
NEWS in-hospital mortality

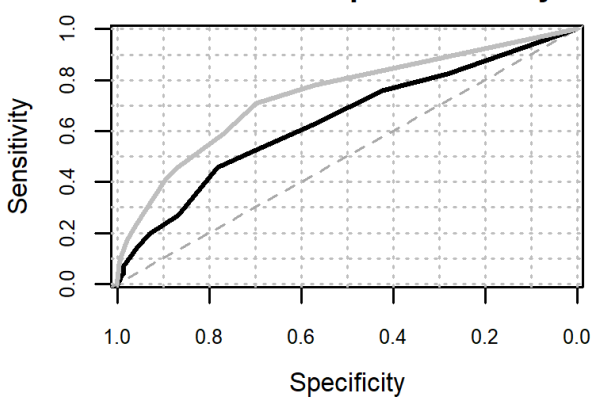

NEWS within 24 hour mortality

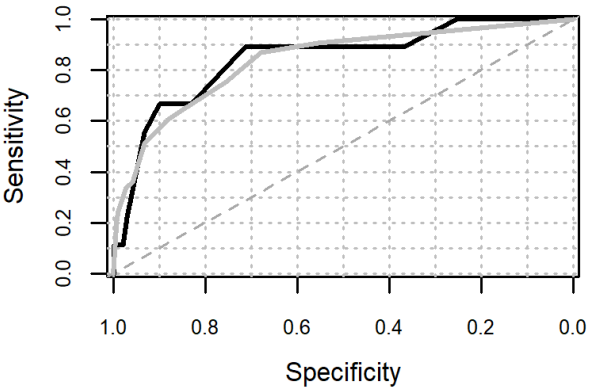

NEWS within 48 hour mortality

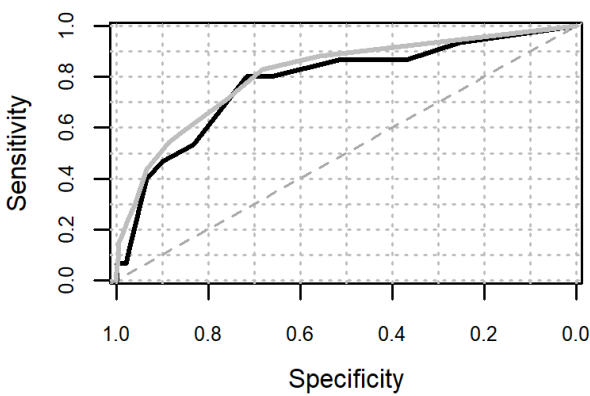

NEWS within 72 hour mortality

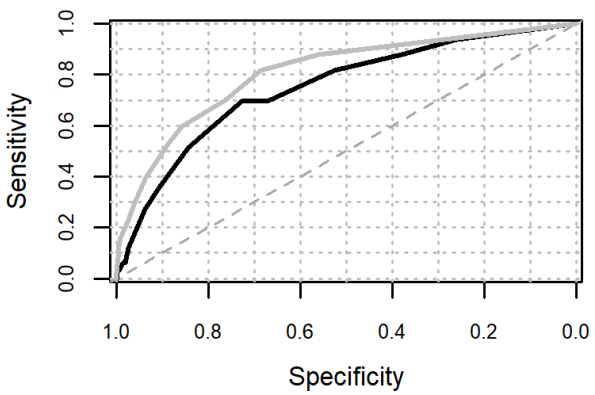

NEWS2 in-hospital mortality

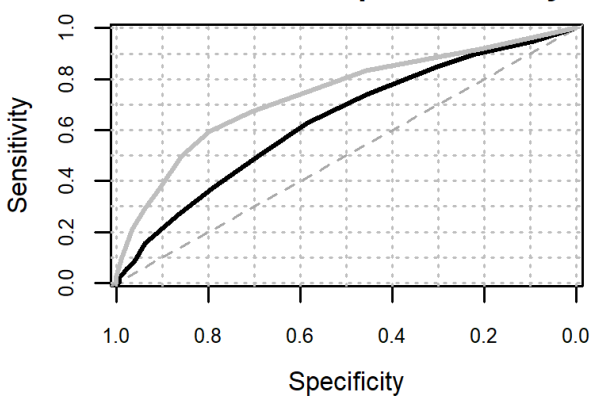

NEWS2 within 24 hour mortality

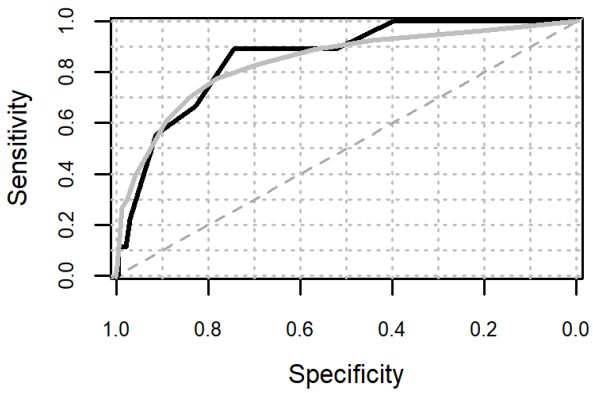

NEWS2 within 48 hour mortality

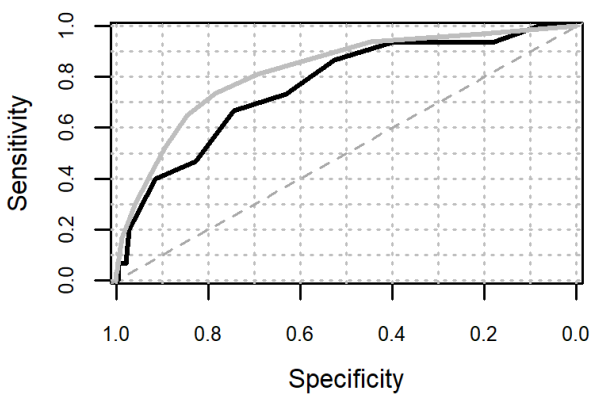

NEWS2 within $\mathbf{7 2}$ hour mortality

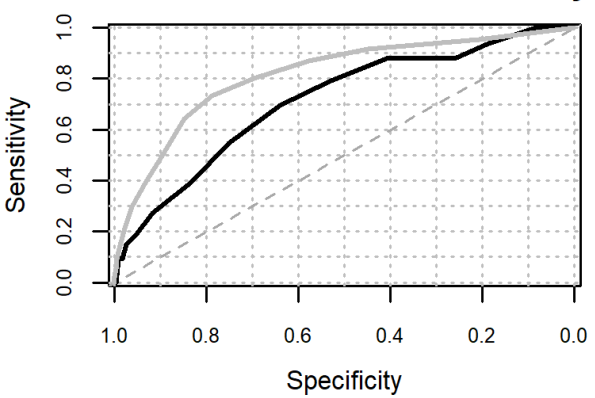

Figure 2 Receiver operating characteristic curve for NEWS2 and NEWS in predicting the risk of in-hospital mortality, mortality within 24 hours, 48 hours and 72 hours in the COVID-19 (black colour) and non-COVID-19 (grey colour) admissions. NEWS, National Early Warning Score.

A recent paper by Kostakis $e t a l^{7}$ found good discrimination for NEWS or NEWS2 (c-statistics 0.842-0.894) concluding that their results 'support the national and international recommendations for the use of NEWS or NEWS2 for the assessment of acute-illness severity in patients with COVID-19.' In contrast to our approach, Kostakis et $a l^{7}$ used the last or ultimate recorded NEWS2/ NEWS within 24 hours of death or ICU admission. We note that when we consider death within 24 hours of admission, our reported c-statistics for index NEWS/ NEWS2 are comparable with those of Kostakis et al. ${ }^{7}$

So taken together these findings indicate that care must be taken not to interpret the predictive power of the ultimate NEWS or NEWS2 score (taken within 24 hours of death) as being equivalent to the predictive power of the index NEWS or NEWS2 score (or preceding NEWS or NEWS2 scores) for risk of in-hospital mortality. The ultimate NEWS or NEWS2 is an accurate predictor of 


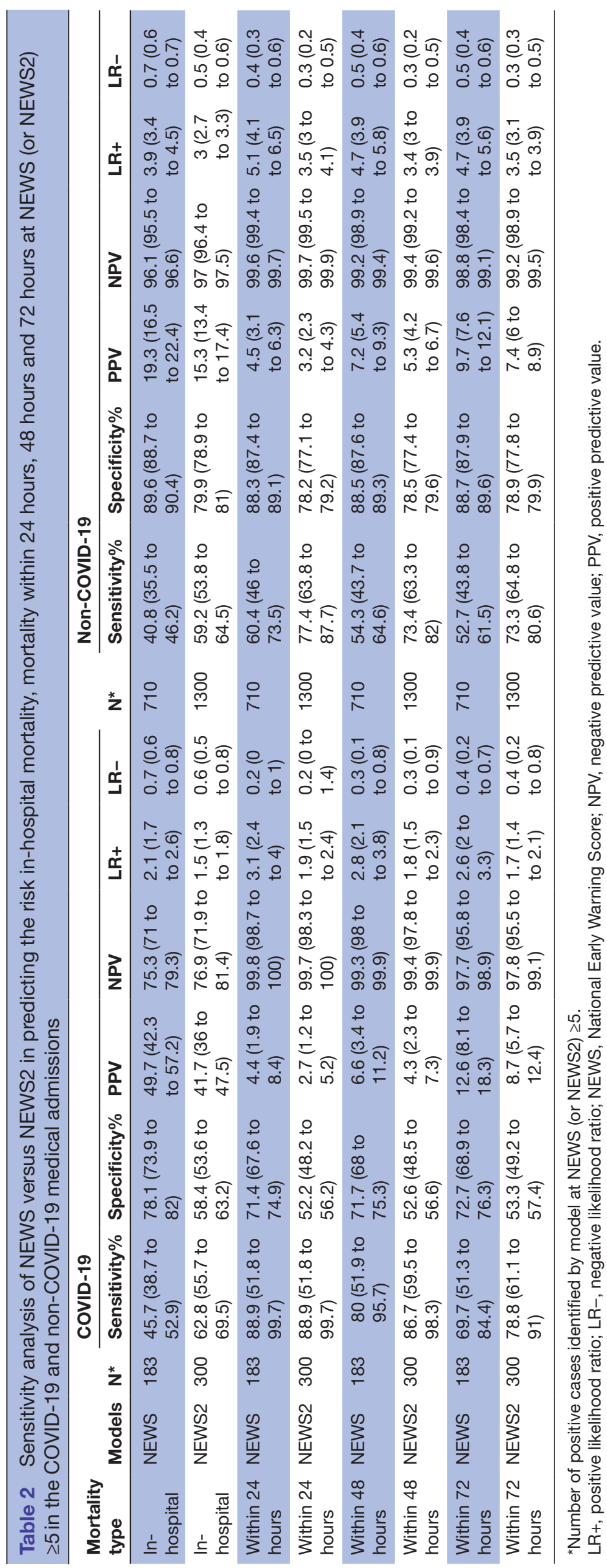


mortality (plus ICU admission in the case of Kostakis et al) for COVID-19 patients but offers a maximum of 24hours for appropriate interventions. This good performance is less surprising when we note that, with the exception of patients who are characterised by abnormal physiology (patients recovering from end-stage renal failure or patients recovering from brain injury), 'Patients die not from their disease but from the disordered physiology caused by the disease'. ${ }^{17}$ But, as our findings show, the performance of the index NEWS or index NEWS2 for predicting death in hospital, which offers an early window of opportunity for assessment and intervention, is poorer especially for COVID-19 patients. This needs to be brought to the attention of medical and nursing staff and reflected in escalation protocols and guidelines (which have always highlighted the importance of clinical judgement) to mitigate potential threats to patient safety by promoting situational awareness about the actual, on admission, in-hospital mortality risk for COVID-19 patients.

WHO describes the range of symptoms seen in COVID-19 which include (but are not limited to) dyspnoea, reduced alertness, delirium, fever, tachypnoea and hypoxia (as a common sign in moderate to severe disease). These symptoms are included in the physiological observation set underpinning NEWS and NEWS2 and were more frequent in our COVID-19 patients compared with non-COVID-19 patients. We also found evidence of lower blood pressure and a higher pulse rate in COVID-19 patients. The NEWS2 guidelines ${ }^{8}$ do note that patients with COVID-19 can develop 'silent hypoxia' where oxygen saturations can drop to low levels and precipitate acute respiratory failure quickly without the presence of obvious symptoms of respiratory distress. As such any patients admitted and on supplemental oxygen may develop a rapidly increasing oxygen requirement that may not increase the NEWS2 score. It is stressed that any increase in oxygen requirement should trigger an escalation for review by a competent senior decision-maker. ${ }^{8}$

Consideration should be also be given to enhancing NEWS or NEWS2 so that they can be used in COVID-19 and non-COVID-19 patients rather than needing to change scoring systems or adjust estimations of risk dependent on diagnosis. We have previously demonstrated how a fully automated computer-enhanced NEWS score can be developed which requires no additional data collection and builds on the standardisation provided by NEWS. ${ }^{18}$ We now propose to extend this to include COVID-19 status.

There are several limitations to our study: (1) This study data is from a single NHS Trust and the extent to which these findings are generalisable, especially to minority ethnic groups with higher COVID-19 mortality, needs further study; (2) We used the index NEWS2 which reflects the 'on-admission' risk of mortality of the patients. Nonetheless, NEWS2 is repeatedly updated for each patient according to local hospital protocols, and the extent to which changes in NEWS2 over time reflect changes in mortality risk needs further study and (3) Although we found no evidence of NEWS2 as having a superior performance to NEWS, it is important to note that our index NEWS data are hypothetical in the sense that the Trust has been using NEWS2 since April 2019. Nevertheless, it is worth noting that a recent, although small Italian study based on 71 hospitalised COVID-19 patients found NEWS2 to be a good predictor (with a high c-statistic 0.90) of subsequent ICU admission for COVID-19 patients but was not able to consider mortality because of insufficient events. ${ }^{6}$ Our study did not consider ICU admissions as an outcome because the number of ICU admissions were low but Kostakis $e t a l^{7}$ used it as a composite outcome with in-hospital mortality. ${ }^{5}$

\section{CONCLUSIONS}

The index or on-admission NEWS and NEWS2 offer lower discrimination for COVID-19 admissions versus non-COVID-19 admissions. The index NEWS2 is not better than the index NEWS. For each value of the index NEWS/NEWS2, COVID-19 admissions had a substantially higher risk of mortality than non-COVID-19 admissions which reflects the increased baseline mortality risk of COVID-19.

\section{Author affiliations}

${ }^{1}$ Renal Medicine, York Teaching Hospital NHS Foundation Trust, York, UK

${ }^{2}$ Faculty of Health Studies, University of Bradford, Bradford, UK

${ }^{3}$ Bradford Institute for Health Research, Bradford, UK

${ }^{4}$ NIHR Yorkshire and Humber Patient Safety Translational Research Centre (YHPSTRC), Bradford, UK

${ }^{5}$ Wolfson Centre for Applied Health Research, Bradford, UK

${ }^{6}$ York Teaching Hospital NHS Foundation Trust, York, UK

${ }^{7}$ The Strategy Unit, NHS Midlands and Lancashire Commissioning Support Unit, West Bromwich, UK

\section{Twitter Donald Richardson @dzrichar}

Contributors DR and MM had the original idea for this work. MFa undertook the statistical analyses with guidance from MM. MFi and KB extracted the necessary data frames. DR gave a clinical perspective. DR, MFa and MM wrote the first draft of this paper and all authors subsequently assisted in redrafting and have approved the final version.

Funding This research was supported by the Health Foundation. The Health Foundation is an independent charity working to improve the quality of health care in the UK. This research was supported by the National Institute for Health Research (NIHR) Yorkshire and Humberside Patient Safety Translational Research Centre (NIHR YHPSTRC).

Disclaimer The views expressed in this article are those of the author(s) and not necessarily those of the NHS, the NIHR, or the Department of Health and Social Care.

Competing interests None declared.

Patient consent for publication Not required.

Ethics approval This study used anonymised data and received ethical approval from the Health Research Authority (HRA) and Health and Care Research Wales (HCRW) (reference number 19/HRA/0548).

Provenance and peer review Not commissioned; externally peer reviewed.

Data availability statement Data may be obtained from a third party and are not publicly available. Our data sharing agreement with NHS York hospital trust 
does not permit us to share this data with other parties. Nonetheless if anyone is interested in the data, then they should contact the R\&D offices in the first instance.

Supplemental material This content has been supplied by the author(s). It has not been vetted by BMJ Publishing Group Limited (BMJ) and may not have been peer-reviewed. Any opinions or recommendations discussed are solely those of the author(s) and are not endorsed by BMJ. BMJ disclaims all liability and responsibility arising from any reliance placed on the content. Where the content includes any translated material, BMJ does not warrant the accuracy and reliability of the translations (including but not limited to local regulations, clinical guidelines, terminology, drug names and drug dosages), and is not responsible for any error and/or omissions arising from translation and adaptation or otherwise.

Open access This is an open access article distributed in accordance with the Creative Commons Attribution Non Commercial (CC BY-NC 4.0) license, which permits others to distribute, remix, adapt, build upon this work non-commercially, and license their derivative works on different terms, provided the original work is properly cited, appropriate credit is given, any changes made indicated, and the use is non-commercial. See: http://creativecommons.org/licenses/by-nc/4.0/.

ORCID iD

Muhammad Faisal http://orcid.org/0000-0003-4885-4251

\section{REFERENCES}

1 Gorbalenya AE, Baker SC, Baric RS, et al. The species severe acute respiratory syndrome-related coronavirus: classifying 2019-nCoV and naming it SARS-CoV-2. Nat Microbiol 2020;5:536-44.

2 Onder G, Rezza G, Brusaferro S. Case-Fatality rate and characteristics of patients dying in relation to COVID-19 in Italy. JAMA 2020;323:1775-6.

3 Vincent J-L, Taccone FS. Understanding pathways to death in patients with COVID-19. Lancet Respir Med 2020;8:430-2.

4 Royal College of Physicians. National Early Warning Score (NEWS): Standardising the assessment of acuteillness severity in the NHS Report of a working party, 2012.

5 NHS. Royal College of physicians: NHS England approves use of national early warning score (news) 2 to improve detection of acutely ill patients, 2017. Available: https://www.rcplondon.ac.uk/news/ nhs-england-approves-use-national-early-warning-score-news-2improve-detection-acutely-ill
6 Gidari A, De Socio GV, Sabbatini S, et al. Predictive value of national early warning score 2 (NEWS2) for intensive care unit admission in patients with SARS-CoV-2 infection. Infect Dis 2020;52:698-704.

7 Kostakis I, Smith GB, Prytherch D, et al. The performance of the National early warning score and national early warning score 2 in hospitalised patients infected by the severe acute respiratory syndrome coronavirus 2 (SARS-CoV-2). Resuscitation 2021;159:150-7.

8 RCP London. NEWS2 and deterioration in COVID-19. Available: https://www.rcplondon.ac.uk/news/news2-and-deterioration-covid19 [Accessed 24 Jun 2020].

9 WHO. ICD-10 : international statistical classification of diseases and related health problems : tenth revision. Available: https://apps.who. int/iris/handle/10665/42980

10 Jolley RJ, Quan H, Jetté N, et al. Validation and optimisation of an ICD-10-coded case definition for sepsis using administrative health data. BMJ Open 2015;5:e009487.

11 Steyerberg EW, Vickers AJ, Cook NR, et al. Assessing the performance of prediction models: a framework for traditional and novel measures. Epidemiology 2010;21:128-38.

12 Hanley JA, McNeil BJ. The meaning and use of the area under a receiver operating characteristic (ROC) curve. Radiology 1982;143:29-36.

13 Robin X, Turck N, Hainard A, et al. pROC: an open-source package for $\mathrm{R}$ and $\mathrm{S}+$ to analyze and compare ROC curves. BMC Bioinformatics 2011;12:77.

14 R Development Core Team. R: A language and environment for statistical computing. R foundation for statistical computing, 2015. Available: http://www.r-project.org/

15 von Elm E, Altman DG, Egger M, et al. The strengthening the reporting of observational studies in epidemiology (STROBE) statement: guidelines for reporting observational studies. PLoS Med 2007; 4:e296.

16 StatCorp. Stata: release 14. statistical software. College Station: StataCorp LP, 2016.

17 McGinley A, Pearse RM. A national early warning score for acutely ill patients. BMJ 2012;345:e5310.

18 Faisal M, Richardson D, Scally A, et al. Performance of externally validated enhanced computer-aided versions of the National early warning score in predicting mortality following an emergency admission to hospital in England: a cross-sectional study. BMJ Open 2019;9:e031596. 